\title{
Perceptual responses in patients with inflammatory and functional bowel disease
} L Chang, J Munakata, E A Mayer, M J Schmulson, T D Johnson, C N Bernstein, L Saba,
B Naliboff, P A Anton, K Matin
UCLA/CURE

Neuroenteric Disease Program, Department of Medicine and

Physiology, UCLA

School of Medicine,

Los Angeles,

California, USA

L Chang

J Munakata

E A Mayer

M J Schmulson

L Saba

B Naliboff

P A Anton

K Matin

Harbor-UCLA Inflammatory Bowel

Disease Center,

Harbor-UCLA

Medical Center,

Torrance, California,

USA

L Chang

Department of Biomathematics, UCLA School of

Medicine, Los Angeles, California, USA

T D Johnson

Department of Medicine, University of Manitoba, Winnipeg, Manitoba, Canada

C N Bernstein

Correspondence to: Dr L Chang, UCLA/CURE Neuroenteric Disease Program, West Los Angeles VA Medical Center, Building 115, Room 223, Los Angeles, CA 90073, USA. Email:linchang@ucla.edu

Accepted for publication 4 April 2000

\begin{abstract}
Background and aims-Enhanced visceral sensitivity following a transient inflammatory process in the gut has been postulated as an aetiological mechanism of irritable bowel syndrome (IBS). In this study we compared perceptual responses to rectosigmoid distension in patients with mild chronic inflammation of the rectum (ulcerative colitis (UC)) and patients without mucosal inflammation (IBS) to determine if chronic low grade mucosal inflammation may be a plausible explanation for rectosigmoid hypersensitivity reported in both IBS and UC patients.

Methods-UC disease activity was quantified using activity index scores. Perception thresholds for discomfort during rectosigmoid distension were compared between 11 UC patients with quiescent or mild disease activity, 18 IBS patients, and 13 healthy controls.

Results-Although UC activity index scores negatively correlated with perceptual thresholds for discomfort $(r=-0.76$, $\mathbf{p}=0.016$ ), UC patients had higher discomfort thresholds compared with IBS patients and controls before $(p=0.02)$ and after $(p<0.001)$ a noxious sigmoid conditioning stimulus.

Conclusions-Rectal perception was attenuated in UC but enhanced in IBS. In chronic mild inflammation, activation of antinociceptive mechanisms may prevent the development of visceral hyperalgesia. Low grade mucosal inflammation alone is unlikely to be responsible for symptoms in functional gastrointestinal disorders. (Gut 2000;47:497-505)
\end{abstract}

Keywords: ulcerative colitis; irritable bowel syndrome; abdominal pain

Chronic intermittent symptoms of abdominal pain and/or discomfort, urgency, and sensation of incomplete evacuation are characteristic of both ulcerative colitis (UC), a disease involving chronic mucosal inflammation, and of irritable bowel syndrome (IBS), a disorder without detectable mucosal alterations. In both disorders abdominal pain is a common feature during flares but symptoms generally resolve during remission. While sensitisation of afferent pathways innervating the colon are thought to be responsible for symptom generation in inflammatory conditions (that is, UC), the mechanism(s) underlying reported visceral hypersensitivity in IBS patients is poorly understood.
In a subset of IBS patients ("post-infectious IBS"), enhanced visceral sensitivity following a transient inflammatory process in the gut has been postulated as an aetiological mechanism. ${ }^{1}$ Some patients with IBS report the onset of their abdominal symptoms following an acute episode of gastroenteritis, ${ }^{12}$ with symptoms persisting despite clearance of the organism. Those patients who develop IBS symptoms have been found to have a greater number of chronic inflammatory cells in the rectal mucosa than patients who become asymptomatic following acute gastroenteritis, despite similar perception thresholds during rectal distension. ${ }^{3}$

In principal, mucosal inflammation could result in chronic abdominal symptoms by inducing a state of chronic visceral hypersensitivity. There are several possibilities by which such chronic hypersensitivity could develop: (1) transient mucosal inflammation may induce neuroplastic changes of visceral afferent pathways which might outlast the original tissue injury ${ }^{4}$; (2) transient mucosal inflammation may sensitise immune cells within the gut. Following resolution of the inflammatory state, trigger factors such as psychosocial stressors may induce recurrence of the inflammation via activation of sensitised immune cells ${ }^{5}$; (3) low grade chronic inflammation or preinflammatory changes in the mucosa may be associated with chronically sensitised visceral afferent pathways.

A series of studies support the concept that mild chronic inflammation of the intestine is not associated with enhanced mechanosensitivity. While patients with active UC report non-noxious and noxious sensations at smaller distension volumes applied to the rectum than healthy controls, ${ }^{6-8}$ in patients with quiescent colitis, distension volumes were found to be similar to those observed in healthy controls. ${ }^{67}$ Several studies evaluating visceral ${ }^{9}$ and somatic sensitivity ${ }^{10}$ in patients with Crohn's disease presented evidence for reduced pain sensitivity, possibly related to activation of antinociceptive mechanisms in response to ongoing intestinal inflammation.

In the current study, by comparing perceptual responses to rectosigmoid distension between UC patients with mild inflammation of the rectosigmoid and IBS patients, we wished to determine if chronic low grade inflammation limited to the mucosa might be a plausible explanation for rectosigmoid hypersensitivity, reported in both IBS and UC

Abbreviations used in this paper: IBS, irritable bowel syndrome; UC, ulcerative colitis; AI, activity index; DAI, disease activity index; TNBS, trinitrobenzene sulphonic acid. 
patients. Our study differs from previous perception studies in inflammatory bowel disease in several ways: (1) non-biased psychophysiological measures were used; (2) the effect of a noxious sigmoid distension stimulus $^{11}$ on rectal perception was studied; and (3) the relationship between quantitative measures of inflammatory severity and rectal perception was assessed. Specifically, we sought to answer the following questions: (i) is chronic mild mucosal inflammation in UC patients associated with enhanced perceptual responses to rectal and sigmoid mechanical stimulation and (ii) do perceptual responses to rectal balloon distension correlate with disease activity in either IBS or UC?

\section{Methods}

SUBJECTS

Healthy controls

Thirteen healthy control subjects (six women and seven men; mean age 36 years; range 21-67) with no evidence of acute or chronic illness were recruited by newspaper advertisement. In particular, there was no evidence of an acute or chronic pain syndrome or abdominal symptoms by bowel symptom questionnaire, personal history, or physical examination.

\section{UC patients}

Eleven UC patients with inactive or mild disease activity (five women and six men; mean age 45 years; range 24-60) were recruited from the UCLA inflammatory bowel disease clinics. A diagnosis of UC was documented by clinical characteristics, endoscopic appearance, and histology. All UC patients were considered to have quiescent or mildly active disease by Truelove and Witts criteria. ${ }^{12}$ In this study, disease activity in UC patients was defined as "quiescent" if sigmoidoscopy revealed no active inflammation or as "active" if inflammation (for example, friable mucosa, ulcerations, exudation) was present. UC disease activity was also assessed by standardised quantitative measures (activity index (AI) ${ }^{13}$ and disease activity index (DAI)). ${ }^{14} \mathrm{AI}$ is based on clinical and laboratory data while DAI also utilises the physician's global assessment and sigmoidoscopic findings. Three UC patients had a history of pancolitis and eight patients had left sided colitis. All but four patients were receiving 5 -aminosalicylic acid products. The remaining four patients were not receiving maintenance therapy. None of the UC patients in clinical remission met ROME criteria for a diagnosis of IBS.

\section{IBS patients}

Eighteen IBS patients (nine women and nine men; mean age 39 years; range 26-60) were recruited from the UCLA Functional Bowel Disease Clinic and the gastroenterology clinics at the West Los Angeles VA and HarborUCLA Medical Centers. Selection criteria included a positive diagnosis by the ROME criteria, ${ }^{15}$ presence of $\geqslant 3$ Manning criteria, ${ }^{16}$ a clinical diagnosis of IBS made by a gastroenterologist experienced in the diagnosis of func- tional bowel disorders, and exclusion of organic disease.

All UC and IBS patients who met inclusion criteria were asked to take part in the study but approximately $20 \%$ refused to participate. None of the patients withdrew from the study voluntarily or was withdrawn by the investigators. Verbal and written consent was obtained from each subject. The study was approved by the West Los Angeles VA Medical Center Research and Development Committee and Committee on Human Studies.

MATERIALS AND APPARATUS

Bowel symptom questionnaire

All subjects completed a UCLA bowel symptom questionnaire on attending the centre. In addition to abdominal symptoms and bowel habits, additional measures included assessment of acute and chronic symptom severity and evaluation of current gastrointestinal symptom intensity by $20 \mathrm{~cm}$ validated sensory and affective graphic verbal descriptor scales. ${ }^{17}$

\section{Psychological symptom checklist}

All subjects completed the SCL-90 symptom checklist ${ }^{18}$ which assesses current psychological symptom severity in the following areas: anxiety, depression, hostility, interpersonal sensitivity, obsessive-compulsive behaviour, paranoia, phobic behaviour, psychosis, and somatisation.

\section{Visceral stimulation device}

Distension of the sigmoid colon and rectum was achieved by air inflation of a double balloon as previously described in detail. ${ }^{11}$ The use of a computer driven volume displacement device allowed for controlled inflation of the balloons. ${ }^{19} 20$ The distension device was programmed to deliver distension at a volume rate ( $870 \mathrm{ml} / \mathrm{min}$ ) to constant pressure plateaus, to simultaneously record pressures and volumes (sampling rate 1 per second), and to log the sensations (that is, no sensation, moderate sensation, discomfort, and pain) from a hand held push-button marker device on a data file. We have previously validated the response characteristics of the distension device. ${ }^{21}$

The double balloon catheter consisted of two identical latex balloons (external diameter $5 \mathrm{~cm}$; length $9 \mathrm{~cm}$ ) attached to a silastic elastomer tube (external diameter $18 \mathrm{~F}$ ) at both proximal and distal ends (MAK-LA, Los Angeles, California, USA). The distance between the two balloons was $9 \mathrm{~cm}$. Before and after completion of every procedure, each balloon was inflated three times to rule out leaks and measure intrinsic compliance (during the third distension) as previously described. ${ }^{21} 22$ The intrinsic compliance of the latex balloons was electronically subtracted from the rectal and sigmoid compliance values obtained in vivo. $^{21}{ }^{22}$ A flexible sigmoidoscopy (Olympus CF-100S) to $40 \mathrm{~cm}$ from the anal verge was performed without premedication on each subject for placement of the balloons (one rectum; one sigmoid). The severity and extent of disease were documented in UC patients during sigmoidoscopy. Following insertion of 


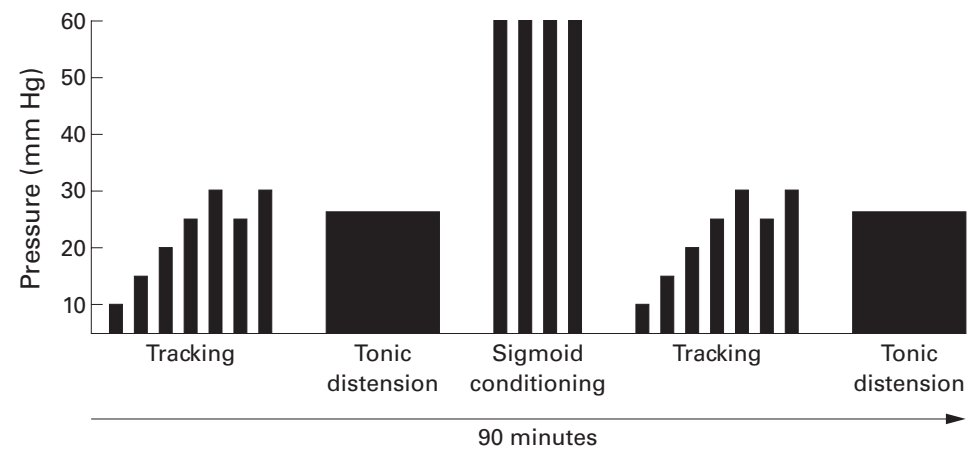

Figure 1 Sequence of rectal and sigmoid distension paradigms. Rectal threshold tracking and tonic distension paradigms were performed before and after the sigmoid conditioning stimulus. This illustration does not represent the actual number of distensions given.

the proximal tip of a Teflon guidewire through the channel of the sigmoidoscope, the sigmoidoscope was withdrawn with evacuation of air. The lubricated double balloon catheter was then passed over the guidewire such that the distal balloon was $4 \mathrm{~cm}$ from the anal verge. The catheter was secured with tape and the wire was withdrawn. Fluoroscopy studies have confirmed correct positioning of the rectosigmoid balloons using this technique (unpublished observations).

EXPERIMENTAL PROTOCOI

All medications known to affect the gastrointestinal tract were discontinued 48 hours before the procedure, with the exception of 5 -aminosalicylic acid products used by six of the UC patients. None of the UC patients was receiving corticosteroids or immunosuppressive agents for at least one month prior to the study. A 12 hour fast and application of two Fleets enemas (CB Fleet Co., Inc., Lynchburg, Virginia, USA) preceded sigmoidoscopy for balloon placement. All experimental rectosigmoid stimulation studies were performed 30 minutes after balloon placement. Subjects were placed in the left lateral decubitus position on a padded table. In UC patients, $10 \mathrm{ml}$ of venous blood were obtained for measurement of haematocrit, erythrocyte sedimentation rate, and serum albumin. ${ }^{13}$

Although the examiner was always present, interaction with the subjects ceased after initial explanation of the respective task. Subjects had no visual or auditory cues to anticipate the location or time courses of the distensions, nor were they instructed about the nature of the distension protocols. Two distension protocols, sensory tracking and tonic stimulus, were used to evaluate rectal perception during baseline and were repeated following sigmoid stimulation. Baseline does not refer to true resting conditions as perceptual responses may be influenced by autonomic responses to the presence of the rectosigmoid balloon. The sequence of the rectal and sigmoid distensions is illustrated in fig 1 .

\section{Threshold tracking paradigm (rectum)}

To obtain a measure of rectal sensitivity, rectal perception was assessed by measuring discomfort thresholds in response to a non-biased rectal phasic distension paradigm (rectal sensory tracking). The electronic distension device was programmed to deliver intermittent phasic stimuli (30 second duration; $5 \mathrm{~mm} \mathrm{Hg}$ increments) separated by an interpulse interval (30 second duration; $5 \mathrm{~mm} \mathrm{Hg}$ ) within a nonbiased stimulus tracking paradigm, as previously reported by Whitehead and colleagues. ${ }^{23}$ Total duration of the sensory tracking paradigm was 600 seconds. All patients and controls completed the entire number of distension trials. During each stimulus and rest, subjects were prompted by the distension device to report the intensity of their sensations by triggering the push-button marker device. If the subject indicated a sensation below the discomfort level (that is, no sensation or moderate sensation), the following stimulus was increased by $5 \mathrm{~mm} \mathrm{Hg}$. If the subject indicated discomfort, the following stimulus was randomised to remain the same or was decreased by $5 \mathrm{~mm} \mathrm{Hg}$. If the subject reported the onset of pain, the following stimulus was always decreased by $5 \mathrm{~mm} \mathrm{Hg}$.

Tonic distension (rectum)

To determine if sigmoid stimulation differentially affected perceptual responses to rapid phasic distension and continuous tonic distension, a 180 second stimulus was used. Individual stimulus levels were set at the pressure of the subject's discomfort threshold during the sensory tracking protocol.

\section{Noxious distension (sigmoid colon)}

To determine the effect of a noxious mechanical sigmoid colon stimulus on perception of rectal distension, subjects received repetitive distension of the sigmoid colon over a period of 600 seconds. During this period, the sigmoid received intermittent rapid phasic distensions (30 seconds duration; $60 \mathrm{~mm} \mathrm{Hg}$ ) with an interpulse rest ( 30 seconds duration; $5 \mathrm{~mm} \mathrm{Hg}$ ); $25 \%$ of UC patients, $28 \%$ of IBS patients, and $31 \%$ of healthy controls were unable to complete the entire task. The mean number of distensions tolerated was not different between UC patients, IBS patients, and controls.

\section{EVALUATION OF OUTCOME PARAMETERS}

\section{Discomfort thresholds}

Perception thresholds for rectal discomfort and onset of pain were determined from the tracking protocol and expressed with reference to intrarectal pressure and estimated wall tension. Discomfort thresholds were quantified by averaging the last six stimulus pressures of the tracking protocol. The length of the task was 600 seconds which is sufficient to give stable discomfort thresholds. ${ }^{24}$ Wall tension was estimated using an assumed cylindrical shape of the rectal balloon with a length of $9 \mathrm{~cm}$. Even though the shape of the balloon when maximally inflated outside of the patient may not be cylindrical, the estimate may be adequate for partial inflations of the balloon against the resistance of the bowel wall. The radius at each discomfort threshold $(\mathrm{mm} \mathrm{Hg})$ was derived from the volume of a cylinder $\left(\mathrm{V}=\pi \mathrm{r}^{2} \mathrm{~L}\right.$, where $\mathrm{L}=9 \mathrm{~cm})$. The wall tension equation applied the estimated balloon radius and the derived 
Table 1 Clinical characteristics of patients with ulcerative colitis (UC)

\begin{tabular}{llllrl}
\hline $\begin{array}{l}\text { UC } \\
\text { patients }\end{array}$ & $\begin{array}{l}\text { Extent of } \\
\text { disease }\end{array}$ & $\begin{array}{l}\text { Duration } \\
\text { of disease }\end{array}$ & Sigmoidoscopic findings & $A I$ & $D A I$ \\
\hline 1 & Left sided & $2 \mathrm{y}$ & Normal & 86 & 1 \\
2 & Left sided & $3 \mathrm{mos}$ & Normal & 125 & 0 \\
3 & Left sided & $3 \mathrm{y}$ & Moderate erythema, friability to $10 \mathrm{~cm}$ & 162 & 5 \\
4 & Left sided & $5 \mathrm{y}$ & Erythema and 2 small rectal ulcers at $8 \mathrm{~cm}$ & 120 & 6 \\
5 & Left sided & $10 \mathrm{y}$ & Moderate friability & 131 & 4 \\
6 & Left sided & $14 \mathrm{y}$ & Moderate erythema and small ulcers to $30 \mathrm{~cm}$ & 91 & 5 \\
7 & Left sided & $20 \mathrm{y}$ & Rectal ulcers & 105.5 & 0 \\
8 & Left sided & $5 \mathrm{y}$ & Normal & 95 & 0 \\
9 & Pancolitis & $17 \mathrm{y}$ & Normal & 112 & 1 \\
10 & Pancolitis & $8 \mathrm{y}$ & Normal & 84 & 0 \\
11 & Pancolitis & $9 \mathrm{y}$ & Normal & 98.6 & 0
\end{tabular}

AI, activity index; DAI, disease activity index.

pressure using LaPlace's law (cylinder, $\mathrm{T}=2 \mathrm{pr})$.

\section{Affective and sensory intensity ratings of distensions}

Subjective unpleasantness of both the rectal sensory tracking and the tonic rectal stimulus tasks were assessed before and after sigmoid stimulation using validated graphic descriptor scales. ${ }^{17}$ The affective scale consisted of descriptors of increasing unpleasantness ranging from "none" to "very intolerable" arrayed along a vertical $20 \mathrm{~cm}$ vertical bar. Ratings were assessed immediately following each task. Subjective unpleasantness ratings were also measured following the noxious sigmoid stimulus by validated graphic descriptor scales. In addition, sensory intensity ratings for pressure were measured during the noxious sigmoid stimulus using a handheld visual scale device where the patient was able to continuously grade intensity of pressure with a scale ranging from 0 ("neutral") to 100 ("severe").

Viscerosomatic referral

Viscerosomatic referral patterns were assessed as a measure of central modulation of viscerosomatic convergence. ${ }^{22}$ At the conclusion of each distension protocol, subjects were asked to mark the location and corresponding extent of their sensations on a body map. A transparent acetate sheet with a replica of the body map and dermatomes was overlaid on subject reports. Extent of referral area was quantified by determining the number of dermatomes encompassed by subject markings.

\section{Mechanoelastic properties of rectosigmoid}

To determine if differences in perceptual stimulus ratings may be secondary to chronic inflammation induced changes in the compliance of the rectosigmoid, we used several previously reported techniques to assess the mechanoelastic properties.

Volume changes during isobaric distension

The rate of relaxation of the rectal and sigmoid colon wall during isobaric distension was estimated by volume changes during rapid distension as described previously. ${ }^{21}$ The rate of active relaxation was quantified by dividing the volume increase during the isobaric phase of the pressure pulse by the corresponding change in time.
Changes in resting volume (at the baseline pressure) were used as an estimate of rectal and sigmoid colon tone in response to repetitive distension. The method of determining resting volume has been described previously. ${ }^{11}$

Rectal compliance was calculated at the point of reaching steady state pressure with respect to the corresponding balloon volume during the tonic rectal stimulus and corrected by subtracting intrinsic balloon compliance as previously reported. ${ }^{22}$

\section{STATISTICAL ANALYSIS}

Group comparisons of the descriptive symptom data were performed using the Wilcoxon rank sum test. ${ }^{25}$ Analysis of the main dependent variables for the sensory studies used the Kruskal-Wallis test (a non-parametric version of a one way ANOVA). ${ }^{25}$ Group comparisons of baseline conditions (pre-conditioning) following the sigmoid conditioning stimulus (postconditioning) and changes (post-pre) were performed. Post hoc pairwise comparisons between groups were performed for significant group differences. A Wilcoxon rank sum test was used for this purpose. The relationships between demographic, disease severity, psychological symptom scales, and discomfort thresholds were evaluated using Spearman's correlation coefficient, which is robust to outliers and influential values. ${ }^{25}$ An $\alpha$ cutoff of $\mathrm{p}<0.05$ was used throughout the study. Mean (SEM) values are shown throughout.

\section{Results}

CLINICAL CHARACTERISTICS AND SYMPTOM

SEVERITY

There was no significant difference in age or sex of UC patients compared with IBS patients or healthy controls. Psychological symptom scores were similar between UC patients and both IBS patients and normal subjects. The clinical characteristics of the UC patients are summarised in table 1 . Mean duration of UC was 8.5 (1.9) years (range 4 months to 20 years) and mean time since last disease exacerbation was $1.2(0.4)$ years (range 0.25-5 years).

Five of 11 UC patients experienced intermittent mild abdominal pain during the week prior to their tests. Table 2 shows mean verbal descriptor ratings of clinical gastrointestinal symptoms at the time of the study (acute) and during the previous six months (chronic), measured in patients with UC and IBS. The severity ratings of chronic gastrointestinal symptoms were significantly lower in UC patients compared with IBS patients $(p<0.01)$ while ratings of acute symptoms were similar.

Table 2 Gastrointestinal symptom severity in patients with ulcerative colitis (UC) and irritable bowel syndrom (IBS)

\begin{tabular}{llc}
\hline Gastrointestinal symptom rating & UC patients & IBS patients \\
\hline Acute sensory intensity $(\mathrm{cm})$ & $5(1)$ & $9(2)$ \\
Acute unpleasantness $(\mathrm{cm})$ & $4(1)$ & $7(2)$ \\
Chronic sensory intensity (cm) & $7(1)$ & $15(1)^{\star}$ \\
Chronic unpleasantness (cm) & $7(1)$ & $11(1)^{\star}$ \\
\hline
\end{tabular}

Values are mean (SEM)

${ }^{\star} \mathrm{p}<0.05$ compared with UC patients. 


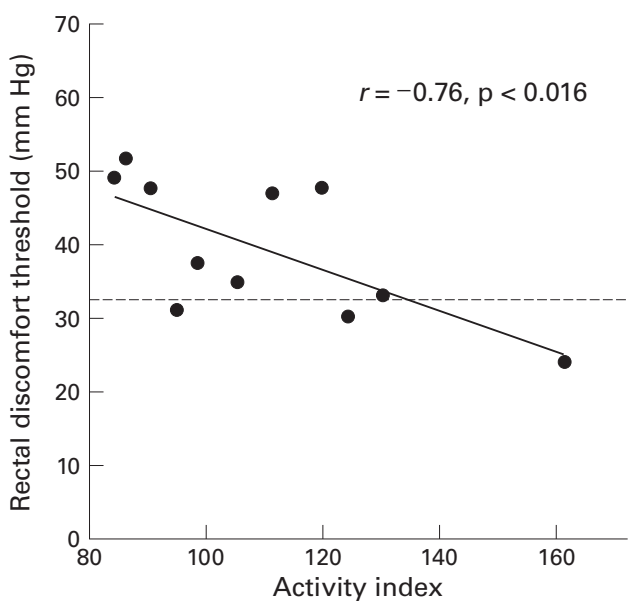

Figure 2 Correlation of ulcerative colitis activity index and rectal discomfort thresholds. Broken line indicates mean rectal discomfort threshold in normal controls.

PERCEPTUAL RESPONSES TO RECTAL DISTENSION Effect of disease activity on perception thresholds in $U C$

The perception of rectal distension was associated with disease activity, as measured by $\mathrm{AI}^{13}$ in patients with UC. Mean discomfort thresholds during rectal distension at baseline significantly inversely correlated with UC AI values $(r=-0.76, \mathrm{p}<0.02$ ) (fig 2 ). AI values and rectal perception were not significantly associated with age, total duration of disease, duration of time since last exacerbation, or verbal descriptor ratings of abdominal discomfort on the day of the study or during the past six months. In contrast, DAI scores ${ }^{14}$ failed to cor-

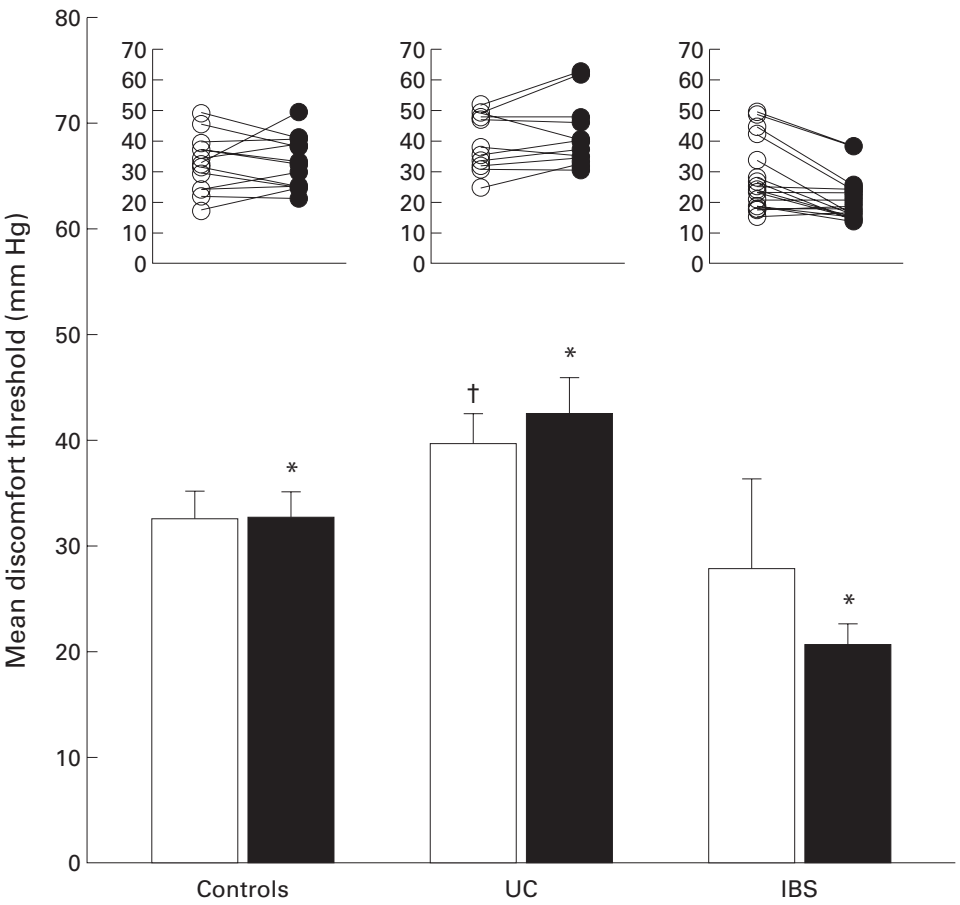

Figure 3 Rectal discomfort thresholds in normal controls, and in patients with ulcerative colitis (UC) and inflammatory bowel disease (IBS) before (open columns) and after (filled columns) noxious sigmoid stimulus. (Bottom) Mean rectal discomfort thresholds are shown; * significant differences compared with the two other groups $(p<0.05)$; tsignificant difference compared with IBS group. (Top) Individual rectal discomfort thresholds. In this and subsequent figures, values are mean (SEM). relate significantly with perceptual thresholds or with the clinical parameters mentioned above.

Differences in perception thresholds between UC, IBS, and healthy control subjects

Significant group effects between UC and both IBS and healthy subjects were observed for discomfort thresholds during rectal distension, regardless of whether the thresholds were expressed in terms of pressure or wall tension (pre-conditioning, $\quad \mathrm{p}=0.016$; postconditioning, $\mathrm{p}<0.001$; change, $\mathrm{p}=0.004)$. As shown in fig 3, mean discomfort thresholds of UC patients were significantly higher than those of IBS patients at baseline (39.6 (2.9) $\mathrm{mm} \mathrm{Hg} v 27.8$ (2.7) $\mathrm{mm} \mathrm{Hg})(\mathrm{p}=0.008)$ and following the noxious sigmoid stimulus $(42.5$ (3.4) $\mathrm{mm} \mathrm{Hg} v 20.7$ (1.8) $\mathrm{mm} \mathrm{Hg})(\mathrm{p}<0.001)$. Six of 11 UC patients developed higher rectal discomfort thresholds following sigmoid stimulation. While the mean discomfort threshold to rectal distension in the UC group remained essentially unchanged $(p=0.21)$ in response to sigmoid stimulation, it decreased significantly in the IBS group $(p=0.003)$. Patients with UC had similar rectal discomfort thresholds compared with controls at baseline (39.6 (2.9) $\mathrm{mm} \mathrm{Hg} v 32.5$ (2.6) $\mathrm{mm} \mathrm{Hg}$; $\mathrm{p}=0.11$ ) but higher thresholds following sigmoid stimulation (42.5 (3.4) $\mathrm{mm} \mathrm{Hg} v 32.7$ (2.4) $\mathrm{mm} \mathrm{Hg})(\mathrm{p}=0.047)$. There were no significant differences between discomfort thresholds in UC patients with mildly active disease and those with quiescent disease.

UNPLEASANTNESS RATINGS OF RECTAL DISTENSION

Phasic distension

In agreement with the higher discomfort thresholds reached in UC subjects, they had lower unpleasantness ratings during phasic rectal distension compared with patients with IBS before $(8.3(0.9) v 11.1(0.7) ; \mathrm{p}=0.048)$ and after $(8.9(0.7) v 11.0(0.7) ; \mathrm{p}=0.07)$ the noxious sigmoid conditioning stimulus. The subjective unpleasantness ratings of rectal perception experienced during phasic rectal distension were similar in controls compared with IBS patients before $(9.1(0.5) v 11.1(0.7)$; $\mathrm{p}=0.27)$ and after (8.5 (0.6) v 11.0 (0.7); $\mathrm{p}=0.02)$ the noxious sigmoid stimulation. There were no significant differences in unpleasantness ratings between UC patients and controls. There was also no significant effect of the noxious sigmoid stimulus on unpleasantness ratings during phasic rectal distension in all three groups.

Tonic rectal distension

Despite receiving higher tonic stimulus pressures, which were set at individual discomfort thresholds, patients with UC had similar unpleasantness ratings during baseline conditions and after sigmoid stimulation compared with the two other groups. There was no significant effect of sigmoid stimulation within each group for sensory ratings. 


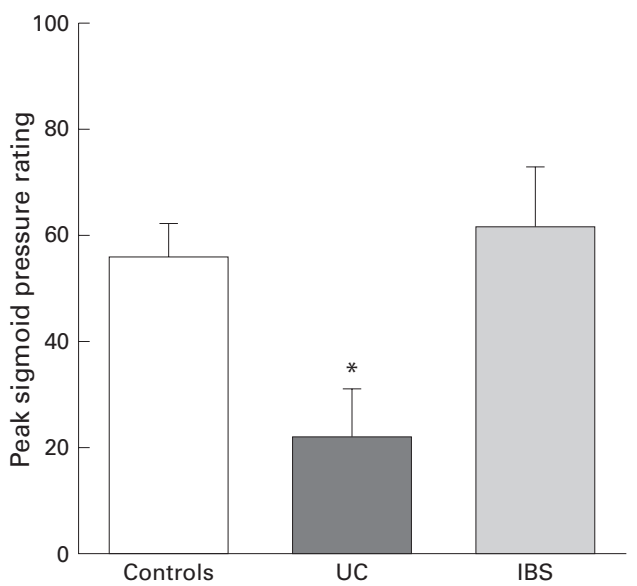

Figure 4 Mean peak sensory ratings for perception of pressure during the initial pressure pulse of the noxious sigmoid stimulus in normal subjects, and in patients with ulcerative colitis (UC) and inflammatory bowel disease (IBS). *Significant difference compared with the two other groups $(p<0.05)$.

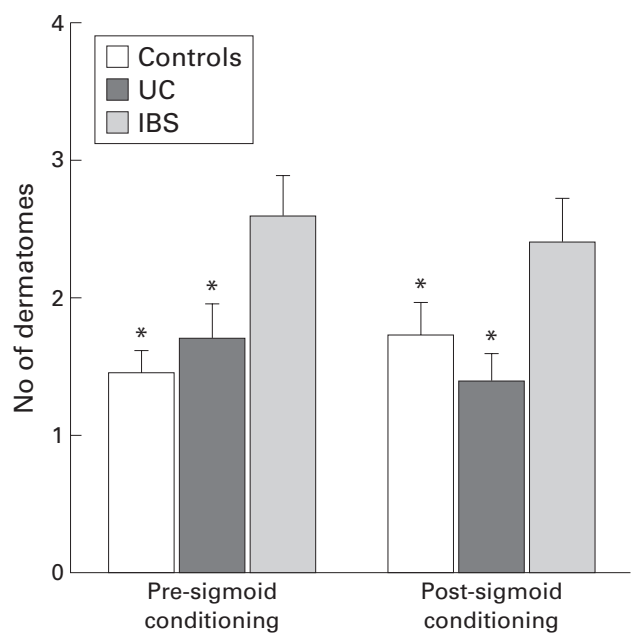

Figure 5 Viscerosomatic referral areas to which healthy subjects and patients with ulcerative colitis (UC) and inflammatory bowel disease (IBS) referred their sensations in response to the tonic rectal stimulus before (left) and after (right) noxious sigmoid stimulus. *Significant difference compared with patients with IBS $(p<0.05)$.

Tolerance and intensity ratings of sigmoid distensions

While all 11 UC patients received intermittent rapid sigmoid distensions of $60 \mathrm{~mm} \mathrm{Hg}$, three of the control individuals (23\%) and five of the IBS patients $(28 \%)$ were unable to tolerate the $60 \mathrm{~mm} \mathrm{Hg}$ stimulation and received pressure pulses of either 40 or $50 \mathrm{~mm} \mathrm{Hg} \mathrm{(not}$ significant). The mean peak sensory rating for perception of pressure using the hand held visual scale device during the initial sigmoid

Table 3 Rectal and sigmoid tone and compliance in patients with ulcerative colitis (UC) and irritable bowel syndrome (IBS) and in healthy controls

\begin{tabular}{llrl}
\hline & UC patients & IBS patients & Controls \\
\hline Rectal resting volume (ml/distension) & & & \\
$\quad$ Baseline & $3.1(0.7)$ & $5.3(1.2)$ & $3.2(0.7)$ \\
$\quad$ Post-sigmoid stimulation & $3.2(1.1)$ & $4.0(0.9)$ & $3.6(0.8)$ \\
Rectal compliance (ml/mm Hg) & & & \\
$\quad$ Baseline & $2.9(0.4)$ & $2.2(0.3)$ & $2.9(0.3)$ \\
$\quad$ Post-sigmoid stimulation & $2.9(0.4)$ & $2.2(0.3)$ & $3.3(0.4)$ \\
Sigmoid resting volume (ml/distension) & $8.1(3.2)$ & $17.2(3.8)$ & $8.2(1.4)$ \\
Sigmoid compliance $(\mathrm{ml} / \mathrm{mm} \mathrm{Hg})$ & $2.7(0.4)$ & $1.9(0.2)$ & $2.3(0.4)$ \\
\hline
\end{tabular}

Values are mean (SEM). pressure pulse was significantly lower in UC patients compared with healthy individuals and IBS patients $(21.9(9.1) v 56.0(6.3)$ and 61.5 (11.1), respectively) $(\mathrm{p}=0.02)$ (fig 4). In addition, the graphic descriptor scales for unpleasantness ratings measured after sigmoid stimulation demonstrated that patients with UC had significantly lower ratings compared with patients with IBS $(p<0.05)$.

Combining patients with UC and IBS and normal subjects, sensory ratings for the noxious sigmoid stimulus negatively correlated with rectal discomfort thresholds. The peak sensory ratings obtained using a 100 point visual scale device during the initial sigmoid pressure pulse significantly correlated with baseline rectal discomfort thresholds $(r=-0.78)(p<0.001)$. Baseline rectal discomfort thresholds also negatively correlated with unpleasantness descriptor ratings during sigmoid distension $(r=-0.70) \quad(p<0.001)$. Not surprisingly, the two methods of assessing sensations experienced during sigmoid distension significantly correlated with each other. The peak sensory ratings of the initial sigmoid pressure pulse positively correlated with the sensory intensity ratings $(r=0.73)(\mathrm{p}<0.001)$.

Viscerosomatic referral

UC subjects reported a lower number of dermatomes to which they referred their sensations than IBS patients during the phasic rectal distension at baseline $(1.7(0.3) v 2.6$ (0.3)) and post-noxious sigmoid stimulation $(1.4(0.2) v 2.4(0.3))(\mathrm{p}=0.005)$. There was no difference between controls and UC patients (fig 5). IBS patients reported a higher number of viscerosomatic referral areas before and after the noxious sigmoid stimulus compared with controls $(p=0.03)$. There was no effect of sigmoid stimulation on the viscerosomatic referral areas within each group.

EFFECT OF THE NOXIOUS SIGMOID STIMULUS ON MECHANOELASTIC PROPERTIES OF THE RECTUM AND SIGMOID

Rectum

Rectal volume at baseline gradually increased during the rectal sensory tracking stimulus in all groups. Changes in resting volume at baseline pressure, compliance, and rate of relaxation during isobaric distension were similar between UC patients, IBS patients, and controls, and were not significantly affected by the noxious sigmoid stimulus (table 3 ). Rectal compliance did not correlate with measures of disease activity in patients with UC.

\section{Sigmoid}

Sigmoid resting volume was comparable at baseline and increased with increasing number of distensions in a similar manner in patients with UC, IBS, and controls (table 3). Sigmoid compliance was also not different between UC patients and controls or between UC and IBS patients (table 3). Sigmoid relaxation rates during isobaric distension were similar between UC, IBS patients, and controls, and did not change during the noxious sigmoid stimulus. 


\section{Discussion}

Reported gastrointestinal symptoms in UC can be indistinguishable from those reported by patients with functional bowel disease, including abdominal discomfort, bloating, sensation of gas and abdominal distension, and sensation of incomplete evacuation following a bowel movement. ${ }^{26}$ However, our current study has demonstrated that patients with inflammatory and functional colonic disease differ in their perceptual responses to rectosigmoid distension. Despite the clinical evidence for mild mucosal inflammation, UC patients had higher discomfort thresholds and lower unpleasantness ratings of rectosigmoid distension, enhanced tolerance of high intensity sigmoid distension, and they referred rectal sensations to a smaller number of dermatomes. While repetitive noxious sigmoid distension induced rectal hyperalgesia in IBS patients, this intervention produced hypoalgesia in more than $50 \%$ of UC patients.

The current study provided several psychophysiological measures consistent with diminished perception of rectal and sigmoid distensions by UC patients. In contrast, the groups did not differ in several measures of rectosigmoid mechanoelastic properties, making it unlikely that perceptual differences in the study population were confounded by inflammation induced smooth muscle changes or fibrosis. These findings are in agreement with those observed by Denis and colleagues ${ }^{27}$ who reported that UC patients with moderately active (contact bleeding) or inactive (no contact bleeding) disease showed no evidence of altered rectal compliance, while UC patients with active colitis with spontaneous bleeding showed loss of rectal distensibility. In this study the authors concluded that the reduced maximal tolerated volume in colitis was probably due to a decrease in rectal distensibility rather than increased sensitivity of inflamed colon. Rao and colleagues ${ }^{6}$ also found that rectal compliance was significantly lower in patients with active colitis compared with patients with quiescent colitis and controls, but values for the latter two groups were similar. A recent report by Gwee demonstrated reduced compliance in patients three months following an acute gastroenteric infection. ${ }^{3}$ In the same study, patients who reported IBS-like symptoms (IBS+) did not differ in compliance changes from those who were symptom free (IBS-), even though the former group had a larger number of chronic inflammatory cells in rectal biopsies. Presumably secondary to reduced rectal compliance in both groups, IBS+ and IBS- individuals reported rectal sensations at similar distension volumes which were smaller than distension volumes observed in control subjects.

Our findings of visceral hyposensitivity in UC patients appear to contrast with reported animal models of acute visceral hyperalgesia involving acute tissue irritation with chemical irritants such as turpentine, acetic acid, formalin, or zymosan. ${ }^{28-30}$ There are also several studies which have shown an enhanced visceromotor response to colorectal distension in rats treated with trinitrobenzene sulphonic acid (TNBS) which is a model of chronic inflammation. ${ }^{31-34}$ However, the tissue injury in these animal models differs in two important aspects from mucosal involvement in UC patients: (1) in animal models the inflammation is frequently transmural ${ }^{35}$ thereby affecting afferent nerve fibres in the outer wall of the bowel; (2) tissue irritation is acute, not allowing the organisms to fully develop slower adaptive changes to attenuate the sensitising effects of the inflammatory changes on visceral afferent pathways. In addition, while TNBS induced colitis is a rat model of chronic inflammation, these distension studies were usually performed within 3-4 days after intrarectal instillation of TNBS, a time when TNBS inflammation is maximal, and therefore it is not analogous to UC patients with mild to quiescent colitis of up to 10 years' duration.

In rats treated with zymosan induced colitis, visceral hyperalgesia mediated by the activity of spinal NMDA and non-NMDA receptors in response to colorectal distension has recently been demonstrated. ${ }^{29}$ The additional finding that afferent fibres from the inflamed colon are no longer sensitised at three hours when hyperalgesia and descending pain modulation systems are active, supports predominant modulation of visceral hyperalgesia associated with visceral inflammation by central mechanisms. ${ }^{36}$

Our finding that chronic mild inflammation of the gut in humans does not result in pathological perception of visceral distension when assessed by rigorous psychophysical techniques makes low grade chronic inflammatory or proinflammatory changes of the mucosa in the absence of associated alterations in antinociceptive mechanisms an unlikely cause of IBS symptoms. These findings are analogous to recent studies by Fass and colleagues ${ }^{37}$ and Mertz and colleagues ${ }^{38}$ who demonstrated that chronic inflammation of the oesophagus and stomach, respectively, are also not associated with visceral mechanical hyperalgesia. Furthermore, evidence for diminished perceptual responses to rectal distension ${ }^{9}$ and to somatic pain ${ }^{10}$ have previously been reported in patients with active Crohn's disease. Bernstein et al demonstrated that Crohn's patients with isolated inflammation in the small bowel have increased discomfort thresholds in the rectum. ${ }^{9}$ This finding demonstrates that chronic inflammation in one part of the bowel is not associated with decreased thresholds of sensory afferent fibres arising from another noninflamed part. Our study differs from the study of Bernstein et al in two main ways: (1) we measured visceral perception at the area of mucosal inflammation and (2) we evaluated the effect of a noxious sigmoid stimulus on rectal perception. The current study was an important sequel to the study by Bernstein et al because we sought to determine if the presence of mild or quiescent inflammation could be directly associated with altered visceral perception. Our findings are also in agreement with results from previous studies which failed to detect significant differences in perceptual 
responses to rectal distension between patients with quiescent UC and healthy control subjects. ${ }^{89}$ In Gwee's study, it is difficult to assess if there were true changes in visceral sensitivity three months following acute gastroenteritis as only volume thresholds were reported and rectal compliance was lower in patients compared with controls. ${ }^{3}$

In all reported studies, perception of rectal distension was measured as the lowest rectal volume that could be perceived, the volume required to induce an urge to defecate, and the maximum tolerated volume to balloon distension. The rectal tracking paradigm used in the present study requires subjects to judge a series of similar but non-identical and unpredictable pressure stimuli, thereby avoiding response bias. ${ }^{24}$ At the same time, we have provided evidence arguing against a confounding effect of differences in mechanoelastic properties of the rectosigmoid. The methodology used in the current study resulted in the selection of non-biased discomfort thresholds, expressed as pressure and wall tension, based primarily on viscerosensory information. While previous studies have evaluated perceptual responses only under baseline conditions, we have demonstrated that a noxious stimulus delivered to the sigmoid colon induces visceral hyperalgesia in IBS patients but does not affect the elevated discomfort thresholds in UC patients.

We found a negative correlation between AI scores and rectal discomfort thresholds even though six of 11 patients had discomfort thresholds above the normal mean. Previous studies have provided evidence to suggest the presence of rectal hypersensitivity in UC patients with active disease. ${ }^{6839}$ Taken together, these findings suggest that symptoms of pain and discomfort experienced by UC patients during disease exacerbation are partially related to the greater degree of inflammation present during flares resulting in transient sensitisation of afferent pathways. In contrast, the relative paucity of pain in patients with mild disease activity may be explained by activation of counterregulatory antinociceptive systems which produce endogenous analgesia. The lack of correlation of perceptual thresholds with DAI scores suggests that sigmoidoscopic findings may not be as accurate in measuring severity of inflammation as laboratory indices.

Potential limitations of this study include patient selection and small sample size. While it is unlikely in human physiological studies, particularly in those which include invasive tests, that the selected subjects are representative of all patients with the same disease, a process of self selection is difficult to avoid, even if larger samples are studied. However, in the current study, the primary hypotheses did not require that the findings were applicable to all patients with inflammatory or functional bowel disease. We proposed to test the hypothesis that chronic mild mucosal inflammatory changes alter visceral sensitivity in a way that could explain the observed visceral hyperalgesia in functional gastrointestinal disorders and we have shown that this is not the case. With regard to the small sample size, we recognise that non- significant differences may be due to lack of statistical power but the sample size was sufficient to detect a difference in perception threshold between healthy controls, and UC and IBS patients.

In summary, our findings are consistent with an adaptive response of the central nervous system to the presence of chronic visceral injury. Current evidence suggests that acute tissue damage results in transient upregulation of pain sensitivity while persistence of the peripheral irritation is associated with activation of counterregulatory mechanisms. These mechanisms include peripheral opioid mediated mechanisms, decreasing the excitability of nociceptors, ${ }^{40}$ descending, bulbospinal pain inhibitory systems decreasing the excitability of dorsal horn neurones, ${ }^{4}$ and modulation of the sensory experience by ascending attentional systems. ${ }^{41}$ The fact that abdominal pain is not a prominent clinical feature in the majority of UC patients with mild disease and that most patients become asymptomatic once an acute flare subsides is consistent with such counterregulatory mechanisms.

1 Gwee KA, Graham JC, McKendrick MW, et al. Psychometric scores and persistence of irritable bowel after infectious diarrhoea. Lancet 1996;347:150-3.

2 Chaudhary NA, Truelove SC. The irritable colon syndrome. $Q \mathcal{F}$ Med 1962;31:307-22.

3 Gwee KA. The role of psychological and biological factors in postinfective gut dysfunction. Gut 1999;44:400-6.

4 Mayer EA, Gebhart GF. Basic and clinical aspects of visceral hyperalgesia. Gastroenterology 1994;107:271-93.

5 Collins S, Mayer EA, Raybould HE, ed. Basic and clinical aspects of chronic abdominal pain. Amsterdam: Elsevier, 1993:62-70.

6 Rao SSC, Read NW, Davison PA, et al. Anorectal sensitivity and responses to rectal distension in patients with and responses to rectal distension in patien.

7 Loennig-Baucke V, Metcalf AM, Shirazi S. Anorectal manometry in active and quiescent ulcerative colitis. Am $\mathcal{f}$ Gasroenterol 1989;84:892-7.

8 Farthing MJG, Lennard-Jones JE. Sensibility of the rectum to distension and the anorectal distension reflex in ulcerative colitis. Gut 1978;19:492-502.

9 Bernstein CN, Niazi N, Robert M, et al. Rectal afferent function in patients with inflammatory and functional intestinal disorders. Pain 1996;66:151-61.

10 Cook IJ, Van Eeden A, Collins SM. Patients with irritable bowel syndrome have greater pain tolerance than normal subjects. Gastroenterology 1987;93:727-33.

11 Munakata J, Naliboff B, Harraf F, et al. Repetitive sigmoid stimulation induces rectal hyperalgesia in patients with irritable bowel syndrome. Gastroenterology 1997;112:55-63.

12 Truelove SC, Witts LJ. Cortisone in ulcerative colitis. BMF 1955;1:1041-8.

13 Seo M, Okada M, Tsuneyoshi T, et al. Evaluation of disease activity in patients with moderately active ulcerative colitis: comparisons between a new activity index and Truelove and Witts' classification. Am F Gastroenterol 1995;90:175963.

14 Sutherland LR, Martin F, Greer S, et al. 5-Aminosalicylic acid enema in the treatment of distal ulcerative colitis, proctosigmoiditis, and proctitis. Gastroenterology 1987;92: 1894-7.

15 Thompson WG, Creed F, Drossman DA, et al. Functional bowel disease and functional abdominal pain. Gastroenterology Intl 1992;5:75-91.

16 Manning AP, Thompson WD, Heaton KW, et al. Towards positive diagnosis in the irritable bowel syndrome. $B M F$
pand

17 Gracely RH, McGrath P, Dubner R. Ratio scales of sensory and affective verbal pain descriptors. Pain 1978;5:5-18.

18 Derogatis LR. SCL-90R. Administration, scoring and procedures manual - II. Towson, MD: NCS Assessments, 1983.

19 Mertz H, Fass R, Hirsh T, et al. Amitryptiline for functional dyspepsia: effect on symptoms, gastric sensitivity and sleep. Gastroenterology 1995;108:A649.

20 Prior A, Sorial E, Sun W-M, et al. Irritable bowel syndrome: differences between patients who show rectal sensitivity and those who do not. Eur $\mathcal{f}$ Gastroenterol Hepatol 1993;5:343-9.

21 Lembo T, Munakata J, Mertz H, et al. Evidence for the hypersensitivity of lumbar splanchnic afferents in irritable hypersensitivity of lumbar splanchnic afferents in irritab

22 Mertz H, Naliboff B, Munakata J, et al. Altered rectal perception is a biological marker of patients with irritable bowel syndrome. Gastroenterology 1995;109:40-52. 
23 Whitehead WE, Crowell MD, Shone D, et al. Sensitivity to rectal distension: validation of a measurement system. Gasrectal distension: validation of

24 Naliboff BD, Munakata J, Fullerton S, et al. Evidence for two distinct perceptual alterations in irritable bowe syndrome. Gut 1997;41:505-12.

25 Hollander M, Wolfe DA. Nonparametric statistical methods. New York: John Wiley and Sons; 1973.

26 Chang L, Mayer EA, Lembcke B, et al, eds. Systemic manifestations of IBD: The pending challenge for subtle diagnosis and treatment. Amsterdam: Kluwer Academic Publishers, 1998:3-19.

27 Denis P, Colin R, Galmiche JP, et al. Elastic properties of the rectal wall in normal adults and in patients with ulcerative colitis. Gastroenterology 1979;77:45-8.

28 Ness TJ, Gebhart GF. Visceral pain: a review of experimental studies. Pain 1990;41:167-234.

29 Coutinho SV, Meller ST, Gebhart GF. Intracolonic zymosan produces visceral hyperalgesia in the rat that is zymosan produces visceral hyperalgesia in the rat that is
mediated by spinal NMDA and non-NMDA receptors. Brain Res 1996;736:7-15.

30 Miampamba M, Chery-Croze S, Gorry F, et al. InflammaMiampamba M, Chery-Croze S, Gorry F, et al. Inflamma-
tion of the colonic wall induced by formalin as a model of acute visceral pain. Pain 1994;57:327-34.

31 Sengupta JN, Snider A, Su X, et al. Effects of kappa opioids in the inflamed rat colon. Pain 1999;79:175-85.

32 Julia V, Mezzasalma T, Bueno L. Influence of bradykinin in gastrointestinal disorders and visceral pain induced by acute or chronic inflammation in rats. Dig Dis Sci 1995;40 1913-21
33 Goldhill J, Pichat P, Roome N, et al. Effect of mizolastine on visceral sensory afferent sensitivity and inflammation during experimental colitis. Arzneimittelforschung 1998;48: 179-84.

34 Morteau O, Julia V, Eeckhout C, et al. Influence of 5- $\mathrm{HT}_{3}$ receptor antagonists in visceromotor and nociceptive responses to rectal distension before and during experimental colitis in rats. Fundam Clin Pharmacol 1994;8:553-62.

35 Garrison DW, Chandler MJ, Foreman RD. Viscerosomatic convergence onto feline spinal neurons from esophagus, heart and somatic fields: effect of inflammation. Pain 1992; 49:373-82.

36 Coutinho SV, Sengupta JN, Gebhart GF. Sensitized afferents from the inflamed colon are not involved in the maintenance of visceral hyperalgesia. Soc Neurosci Abstr 1997;23:1002.

37 Fass R, Naliboff B, Higa L, et al. Differential effect of chronic esophageal acid exposure on mechano- and chemosensitivity in humans. Gastroenterology 1998;115: 1363-73.

38 Mertz H, Fullerton S, Naliboff B, et al. Symptoms and visceral perception in severe functional and organic dyspepsia. Gut 1998;42:814-22.

39 Loening-Baucke V, Metcalf AM, Shirazi S. Anorectal manometry in active and quiescent ulcerative colitis. Am $\mathcal{f}$ Gastroenterol 1989;84:892-7.

40 Stein C. The control of pain in peripheral tissue by opioids. N Engl f Med 1995;332:1685-90.

41 Dixon NF. Preconscious processing. New York: John Wiley and Sons, 1987 